\title{
Front Matter: Volume 7734
}

, "Front Matter: Volume 7734," Proc. SPIE 7734, Optical and Infrared Interferometry II, 773401 (10 August 2010); doi: 10.1117/12.868169

SPIE Event: SPIE Astronomical Telescopes + Instrumentation, 2010, San Diego, SPIE. California, United States 


\title{
PROCEEDINGS OF SPIE
}

\section{Optical and Infrared Interferometry II}

\author{
William C. Danchi \\ Françoise Delplancke \\ Jayadev K. Rajagopal \\ Editors
}

27 June-2 July 2010

San Diego, California, United States

Sponsored by

SPIE

\section{Cooperating Organizations}

American Astronomical Society (United States) - Association of Universities for Research in Astronomy, Inc. (United States) - Astronomical Society of Japan (Japan) - Atacama Large Millimeter/submillimeter Array • Ball Aerospace \& Technologies Corporation (United States) Canadian Astronomical Society (CASCA) (Canada) • Commissariat à l'Energie Atomique (France) • European Astronomical Society (Switzerland) • ESO-European Organisation for Astronomical Research in the Southern Hemisphere (Germany) • Japan Aerospace Exploration Agency (Japan) • Jet Propulsion Laboratory (United States) • NASA Goddard Space Flight Center (United States) • National Astronomical Observatory Japan (Japan) National Radio Astronomy Observatory - SOFIA-Stratospheric Observatory for Infrared Astronomy (United States) - Thirty Meter Telescope Project (United States) • W. M. Keck Observatory (United States)

Published by

SPIE

Volume 7734

Part One of Two Parts

Proceedings of SPIE, 0277-786X, v. 7734 
The papers included in this volume were part of the technical conference cited on the cover and title page. Papers were selected and subject to review by the editors and conference program committee. Some conference presentations may not be available for publication. The papers published in these proceedings reflect the work and thoughts of the authors and are published herein as submitted. The publisher is not responsible for the validity of the information or for any outcomes resulting from reliance thereon.

Please use the following format to cite material from this book:

Author(s), "Title of Paper," in Optical and Infrared Interferometry II, edited by William C. Danchi, Françoise Delplancke, Jayadev K. Rajagopal, Proceedings of SPIE Vol. 7734 (SPIE, Bellingham, WA, 2010) Article CID Number.

ISSN 0277-786X

ISBN 9780819482242

Published by

SPIE

P.O. Box 10, Bellingham, Washington 98227-0010 USA

Telephone +1 3606763290 (Pacific Time) · Fax +1 3606471445

SPIE.org

Copyright (C) 2010, Society of Photo-Optical Instrumentation Engineers

Copying of material in this book for internal or personal use, or for the internal or personal use of specific clients, beyond the fair use provisions granted by the U.S. Copyright Law is authorized by SPIE subject to payment of copying fees. The Transactional Reporting Service base fee for this volume is $\$ 18.00$ per article (or portion thereof), which should be paid directly to the Copyright Clearance Center (CCC), 222 Rosewood Drive, Danvers, MA 01923. Payment may also be made electronically through CCC Online at copyright.com. Other copying for republication, resale, advertising or promotion, or any form of systematic or multiple reproduction of any material in this book is prohibited except with permission in writing from the publisher. The CCC fee code is 0277-786X/10/\$18.00.

Printed in the United States of America.

Publication of record for individual papers is online in the SPIE Digital Library.

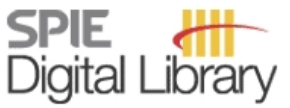

SPIEDigitalLibrary.org

Paper Numbering: Proceedings of SPIE follow an e-First publication model, with papers published first online and then in print and on CD-ROM. Papers are published as they are submitted and meet publication criteria. A unique, consistent, permanent citation identifier (CID) number is assigned to each article at the time of the first publication. Utilization of CIDs allows articles to be fully citable as soon they are published online, and connects the same identifier to all online, print, and electronic versions of the publication. SPIE uses a six-digit CID article numbering system in which:

- The first four digits correspond to the SPIE volume number.

- The last two digits indicate publication order within the volume using a Base 36 numbering system employing both numerals and letters. These two-number sets start with 00, 01, 02, 03, 04, $05,06,07,08,09,0 A, 0 B \ldots 0 Z$, followed by 10-1Z, 20-2Z, etc.

The CID number appears on each page of the manuscript. The complete citation is used on the first page, and an abbreviated version on subsequent pages. Numbers in the index correspond to the last two digits of the six-digit CID number. 


\title{
Contents
}

\section{Part One}

\author{
xxv Conference Committee \\ xxix Unknowns and unknown unknowns: from dark sky to dark matter and dark energy \\ (Plenary Paper) [7733-501] \\ Y. Suto, The Univ. of Tokyo (Japan) \\ xli Optical synoptic telescopes: new science frontiers (Plenary Paper) [7733-502] \\ J. A. Tyson, Univ. of California, Davis (United States)
}

\section{SESSION 1 CURRENT AND PLANNED FACILITIES: GROUND I}

773402 Recent progress at the Keck Interferometer (Invited Paper) [7734-01]

S. Ragland, W. M. Keck Observatory (United States); R. Akeson, NASA Exoplanet Science Institute (United States); M. Colavita, Jet Propulsion Lab. (United States); R. Millan-Gabet, NASA Exoplanet Science Institute (United States); J. Woillez, P. Wizinowich, E. Appleby, B. Berkey, A. Cooper, W. M. Keck Observatory (United States); C. Felizardo, J. Herstein, NASA Exoplanet Science Institute (United States); M. Hrynevych, D. Medeiros, D. Morrison, T. Panteleeva, J.-U. Pott, B. Smith, K. Summers, K. Tsubota, C. Tyau, E. Wetherell, W. M. Keck Observatory (United States)

773403 An update on the CHARA Array (Invited Paper) [7734-02] T. A. ten Brummelaar, H. A. McAlister, CHARA Array, Georgia State Univ. (United States); S. T. Ridgway, National Optical Astronomy Observatory (United States); D. R. Gies, J. Sturmann, L. Sturmann, N. H. Turner, G. H. Schaefer, C. D. Farrington, P. J. Goldfinger, CHARA Array, Georgia State Univ. (United States)

773404 The Very Large Telescope Interferometer: 2010 edition (Invited Paper) [7734-03] P. Haguenauer, J. Alonso, P. Bourget, S. Brillant, P. Gitton, S. Guisard, S. Poupar, N. Schuhler, European Southern Observatory (Chile); R. Abuter, L. Andolfato, European Southern Observatory (Germany); G. Blanchard, J.-P. Berger, A. Cortes, European Southern Observatory (Chile); F. Derie, F. Delplancke, N. Di Lieto, C. Dupuy, European Southern Observatory (Germany); B. Gilli, European Southern Observatory (Chile); A. Glindemann, European Southern Observatory (Germany); S. Guniat, G. Huedepohl, A. Kaufer, J.-B. Le Bouquin, European Southern Observatory (Chile); S. Lévêque, S. Ménardi, European Southern Observatory (Germany); A. Mérand, S. Morel, European Southern Observatory (Chile); I. Percheron, T. Phan Duc, European Southern Observatory (Germany); A. Pino, A. Ramirez, S. Rengaswamy, European Southern Observatory (Chile); A. Richichi, European Southern Observatory (Germany); T. Rivinius, European Southern Observatory (Chile); J. Sahlmann, M. Schoeller, C. Schmid, European Southern Observatory (Germany); S. Stefl, G. Valdes, European Southern Observatory (Chile); G. van Belle, European Southern Observatory (Germany); S. Wehner, European Southern Observatory (Chile); M. Wittkowski, European Southern Observatory (Germany) 
773405 Instrumental developments for the Sydney University Stellar Interferometer (Invited Paper) [7734-04]

J. G. Robertson, M. J. Ireland, W. J. Tango, J. Davis, P. G. Tuthill, A. P. Jacob, Y. Kok, The Univ. of Sydney (Australia); T. A. ten Brummelaar, Georgia State Univ. (United States)

773406 Magdalena Ridge Observatory Interferometer: advancing to first light and new science (Invited Paper) [7734-05]

M. J. Creech-Eakman, V. Romero, I. Payne, New Mexico Institute of Mining and Technology, Magdalena Ridge Observatory (United States); C. Haniff, D. Buscher, Univ. of Cambridge (United Kingdom); C. Aitken, C. Anderson, E. Bakker, T. Coleman, C. Dahl, A. Farris, S. Jiminez, C. Jurgenson, R. King, D. Klinglesmith III, K. McCord, T. McCracken, K. Nyland, A. Olivares, M. Richmond, M. Romero, C. Salcido, J. Sandoval, F. Santoro, J. Seamons, R. Selina, A. Shtromberg, J. Steenson, N. Torres, D. Westpfahl, New Mexico Institute of Mining and Technology, Magdalena Ridge Observatory (United States); F. Baron, M. Fisher, E. Seneta, X. Sun, D. Wilson, J. Young, Univ. of Cambridge (United Kingdom)

773407 Imaging beyond the fringe: an update on the LINC-NIRVANA Fizeau interferometer for the LBT (Invited Paper) [7734-06]

T. M. Herbst, Max-Planck-Institut für Astronomie (Germany); R. Ragazzoni, Vicolo dell'Osservatorio (Italy); A. Eckart, Univ. zu Köln (Germany); G. Weigelt, Max-Planck-Institut für Radioastronomie (Germany)

\section{SESSION 3 CURRENT AND PLANNED FACILITIES: GROUND III}

773408 First results from VLTI near-infrared interferometry on high-mass young stellar objects (Invited Paper) [7734-07]

S. Kraus, Univ. of Michigan (United States); K.-H. Hofmann, K. M. Menten, D. Schertl, G. Weigelt, F. Wyrowski, Max-Planck-Institut für Radioastronomie (Germany); A. Meilland, Max-Planck-Institut für Radioastronomie (Germany) and Lab. Hippolyte Fizeau, Univ. de Nice, Sophia-Antipolis, CNRS, Observatoire de la Cote d'Azur (France); K. Perraut, Lab. d'Astrophysique de Grenoble, Univ. Joseph Fourier, CNRS (France); R. Petrov, S. Robbe-Dubois, Lab. Hippolyte Fizeau, Univ. de Nice, Sophia-Antipolis, CNRS, Observatoire de la Cote d'Azur (France); P. Schilke, I. Physikalisches Institut (Germany); L. Testi, INAF-Osservatorio Astrofisico di Arcetri (Italy)

$773409 \quad$ Mid-infrared interferometry with high spectral resolution (Invited Paper) [7734-08] E. H. Wishnow, W. Mallard, V. Ravi, S. Lockwood, W. Fitelson, D. Wertheimer, C. H. Townes, Univ. of California, Berkeley (United States)

7734 OA Stellar intensity interferometry: astrophysical targets for sub-milliarcsecond imaging [7734-09]

D. Dravins, H. Jensen, Lund Observatory (Sweden); S. LeBohec, P. D. Nuñez, The Univ. of Utah (United States) 
7734 OC Recent science highlights from the Keck Interferometer [7734-11]

R. Akeson, NASA Exoplanet Science Institute (United States); M. Colavita, Jet Propulsion Lab. (United States); R. Millan-Gabet, NASA Exoplanet Science Institute (United States);

S. Ragland, P. Wizinowich, J. Woillez, W. M. Keck Observatory (United States)

7734 OD Performances and first science results with the VEGA/CHARA visible instrument [7734-12]

D. Mourard, OCA, UNS, CNRS FIZEAU (France); M. Tallon, UCBL, CNRS CRAL (France); P. Bério,

D. Bonneau, O. Chesneau, J. M. Clausse, O. Delaa, N. Nardetto, OCA, UNS, CNRS FIZEAU

(France); K. Perraut, UJF, CNRS LAOG (France); A. Spang, P. Stee, OCA, UNS, CNRS FIZEAU

(France); I. Tallon-Bosc, UCBL, CNRS CRAL (France); H. McAlister, T. ten Brummelaar,

J. Sturmann, L. Sturmann, N. Turner, C. Farrington, P. J. Goldfinger, The CHARA Array, Mount Wilson Observatory (United States)

7734 OF Status of PRIMA for the VLTI or the quest for user-friendly fringe tracking [7734-14]

C. Schmid, R. Abuter, S. Ménardi, L. Andolfato, F. Delplancke, F. Derie, N. Di Lieto, R. Frahm, European Southern Observatory (Germany); P. Gitton, European Southern Observatory (Chile); N. Gomes, European Southern Observatory (Germany), SIM - Laboratorio de Sistemas (Portugal), and Univ. do Porto (Portugal); P. Haguenaver, European Southern Observatory (Chile); S. Lévêque, European Southern Observatory (Germany); S. Morel, European Southern Observatory (Chile); A. Müller, European Southern Observatory (Germany) and Max-Planck Institut fur Astronomie (Germany); T. Phan Duc, E. Pozna, European Southern Observatory (Germany); J. Sahlmann, Univ. de Geneve (Switzerland) and European Southern Observatory (Germany); N. Schuhler, European Southern Observatory (Chile); G. van Belle, European Southern Observatory (Germany)

7734 OG MI-6: Michigan interferometry with six telescopes [7734-15]

J. D. Monnier, M. Anderson, F. Baron, D. H. Berger, X. Che, T. Eckhause, S. Kraus, Univ. of Michigan (United States); E. Pedretti, N. Thureau, Univ. of St. Andrews (United Kingdom); R. Millan-Gabet, California Institute of Technology (United States); T. ten Brummelaar, The CHARA Array, Georgia State Univ. (United States); P. Irwin, Consultant (United States); M. Zhao, Jet Propulsion Lab. (United States)

SESSION $5 \quad$ CURRENT AND PLANNED FACILITIES AND INSTRUMENTS: SPACE I

$7734 \mathrm{OH} \quad$ SIM Lite Astrometric Observatory progress report (Invited Paper) [7734-16]

J. C. Marr IV, M. Shao, R. Goullioud, Jet Propulsion Lab. (United States)

$7734 \mathrm{Ol}$ Potential of balloon payloads for in flight validation of direct and nulling interferometry concepts [7734-17]

O. Demangeon, M. Ollivier, IAS, Univ Paris-Sud, CNRS (France); J.-M. Le Duigou, Ctr. National d'Etudes Spatiales (France); F. Cassaing, Office National d'Etudes et de Recherches Aéronautiques (France) and Groupement d'Intérêt Scientifique PHASE (France); V. Coudé du Foresto, LESIA Observatoire de Paris (France) and Groupement d'Intérêt Scientifique PHASE (France); D. Mourard, Observatoire de la Côte d'Azur (France); P. Kern, Lab. d'Astrophysique de l'Observatoire de Grenoble (France); T. Lam Trong, J. Evrard, Ctr. National d'Études Spatiales (France); O. Absil, Astroph. extragalactique et Observations Spatiales (France); D. Defrere, Max Planck Institut für Radioastronomie (Germany); B. Lopez, Observatoire de la Côte d'Azur (France) 
7734 OK The Balloon Experimental Twin Telescope for Infrared Interferometry (BETTII) (Invited Paper) [7734-19]

S. Rinehart, NASA Goddard Space Flight Ctr. (United States)

\section{SESSION 6 CURRENT AND PLANNED FACILITIES AND INSTRUMENTS: SPACE II}

7734 OL Direct imaging of Earth-like planets: why we care about exozodis (Invited Paper) [7734-20]

O. Absil, Univ. de Liège (Belgium); D. Defrère, Max-Planck-Institut fur Radioastronomie (Belgium); A. Roberge, NASA Goddard Space Flight Ctr. (United States); J.-C. Augereau, Lab. d'Astrophysique de Grenoble, CNRS, Univ. Joseph Fourier (France); V. Coudé du Foresto, LESIA, CNRS, Observatoire de Paris à Meudon (France); C. Hanot, Univ. de Liège (Belgium); C. Stark, Univ. of Maryland, College Park (United States); J. Surdej, Univ. de Liège (Belgium)

7734 OM The Fourier-Kelvin Stellar Interferometer (FKSI) - infrared detection and characterization of exozodiacal dust to super-Earths: a progress report [7734-21]

W. C. Danchi, R. K. Barry, NASA Goddard Space Flight Ctr. (United States)

7734 ON Progress in the development of MANIC: a monolithic nulling interferometer for characterizing extrasolar environments [7734-22]

B. A. Hicks, T. A. Cook, Boston Univ. (United States); B. F. Lane, Draper Lab. (United States);

S. Chakrabarti, Boston Univ. (United States)

$773400 \quad$ SIM-Lite narrow-angle modeling and processing [7734-23]

D. W. Murphy, M. H. Milman, D. L. Meier, M. Moshir, Jet Propulsion Lab. (United States)

\section{SESSION 7 OBSERVING TECHNIQUES I}

7734 OP First Keck Interferometer measurements in self-phase referencing mode: spatially resolving circum-stellar line emission of $\mathbf{4 8}$ Lib [7734-24]

J.-U. Pott, Max-Planck-Institut für Astronomie (Germany), W. M. Keck Observatory (United States), and Univ. of California, Los Angeles (United States); J. Woillez, S. Ragland, P. L. Wizinowich, Max-Planck-Institut für Astronomie (Germany); J. A. Eisner, Steward Observatory, The Univ. of Arizona (United States); J. D. Monnier, Univ. of Michigan (United States); R. L. Akeson, NASA Exoplanet Science Institute (United States); A. M. Ghez, Univ. of California, Los Angeles (United States); J. R. Graham, Univ. of California, Berkeley (United States); L. A. Hillenbrand, R. Millan-Gabet, NASA Exoplanet Science Institute (United States); E. Appleby, B. Berkey, Max-Planck-Institut für Astronomie (Germany); M. M. Colavita, Jet Propulsion Lab. (United States); A. Cooper, Max-Planck-Institut für Astronomie (Germany); C. Felizardo, J. Herstein, NASA Exoplanet Science Institute (United States); M. Hrynevych, D. Medeiros, D. Morrison, T. Panteleeva, B. Smith, K. Summers, K. Tsubota, C. Tyau, E. Wetherell, Max-Planck-Institut für Astronomie (Germany)

7734 OS Perspectives for the AMBER Beam Combiner [7734-27]

A. Mérand, S. Stefl, P. Bourget, A. Ramirez, F. Patru, P. Haguenauer, S. Brillant, European Southern Observatory (Chile) 
7734 OT Keck Interferometer nuller instrument performance [7734-28]

M. M. Colavita, E. Serabyn, Jet Propulsion Lab. (United States); S. Ragland, W. M. Keck Observatory (United States); R. Millan-Gabet, R. L. Akeson, NASA Exoplanet Science Institute, California Institute of Technology (United States)

7734 OU Phase closure nulling: results from the 2009 campaign [7734-29]

G. Duvert, F. Malbet, A. Chelli, Lab. d'Astrophysique de l'Observatoire de Grenoble. Univ. J. Fourier, CNRS (France); R. Millan-Gabet, NASA Exoplanet Science Institute (United States); J. D. Monnier, Univ. of Michigan (United States); G. H. Schaefer, CHARA Array of Georgia State Univ., Mount Wilson Observatory (United States)

$7734 \mathrm{OV}$ Assembly and integration activities moving toward commissioning of the Magdalena Ridge Observatory Interferometer [7734-30]

E. Bakker, A. Olivares, F. Santoro, I. Payne, R. Selina, D. Klinglesmith III, A. Farris, R. King, C. Jurgenson, J. Seamons, N. Torres, M. Creech-Eakman, K. McCord, New Mexico Institute of Mining and Technology, Magdalena Ridge Observatory (United States)

7734 OW PACMAN: PRIMA astrometric instrument software [7734-31]

R. Abuter, European Southern Observatory (Germany); J. Sahlmann, Observatoire de Genève (Switzerland) and European Southern Observatory (Germany); E. Pozna, European Southern Observatory (Germany)

\section{SESSION 8 FACILITIES-FUTURE: GROUND I}

7734 OX Signatures of strong gravity with GRAVITY [7734-32]

A. Eckart, Univ. zu Köln (Germany) and Max-Planck-Institut fur Radioastronomie (Germany); M. Zamaninasab, Max-Planck-Institut fur Radioastronomie (Germany); C. Straubmeier, S. Fischer, C. Araujo-Hauck, M. Garcia-Marin, M. Wiest, G. Witzel, R. M. Buchholz, N. Sabha, Univ. zu Köln (Germany); K. Muzic, Univ. of Toronto (Canada); F. Eisenhaver, Max-Planck-Institut für extraterrestrische Physik (Germany); T. Paumard, LESIA, CNRS, Observatoire de Paris à Meudon (France); S. Yazici, Univ. zu Köln (Germany); G. Perrin, Lab. d'Etudes Spatiales et d'Instrumentatio (France) and Institut National des Sciences de I'Univers (France); W. Brandner, Max-Planck-Institut fur Astronomie (Germany); K. Perraut, Lab. d'Astrophysique Observatoire de Grenob (France); A. Amorim, Univ. de Lisboa (Portugal); M. Schöller, European Southern Observatory (Germany)

7734 OY GRAVITY: a four telescope beam combiner instrument for the VLTI [7734-33]

S. Gillessen, F. Eisenhauer, Max-Planck-Institut für extraterrestrische Physik (Germany); G. Perrin, LESIA, Observatoire de Paris à Meudon (France) and Groupement d'Intérêt Scientifique PHASE (France); W. Brandner, Max-Planck-Institut für Astronomie (Germany); C. Straubmeier, Univ. zu Köln (Germany); K. Perraut, Lab. d'Astrophysique de l'Observatoire de Grenoble (France); A. Amorim, Univ. de Lisboa (Portugal); M. Schöller, European Southern Observatory (Germany); C. Araujo-Hauck, Univ. zu Köln (Germany); H. Bartko, Max-Planck-Institut für extraterrestrische Physik (Germany); H. Baumeister, Max-Planck-Institut für Astronomie (Germany); J.-P. Berger, Lab. d'Astrophysique de l'Observatoire de Grenoble (France) and European Southern Observatory (Germany); P. Carvas, Univ. de Lisboa (Portugal); F. Cassaing, ONERA/DOTA (France) and Groupement d'Intérêt Scientifique PHASE (France); F. Chapron, E. Choquet, Y. Clenet, C. Collin, LESIA, Observatoire de Paris à Meudon (France) and Groupement d'Intérêt Scientifique PHASE (France); A. Eckart, Univ. zu Köln (Germany); P. Fedou, LESIA, Observatoire de Paris à Meudon (France); S. Fischer, Univ. zu Köln (Germany); E. Gendron, LESIA, Observatoire de 
Paris à Meudon (France) and Groupement d'Intérêt Scientifique PHASE (France); R. Genzel, Max-Planck-Institut für extraterrestrische Physik (Germany); P. Gitton, European Southern Observatory (Germany); F. Gonte, European Southern Observatory (Germany); A. Gräter, Max-Planck-Institut für extraterrestrische Physik (Germany); P. Haguenauer, European Southern Observatory (Germany); M. Haug, Max-Planck-Institut für extraterrestrische Physik (Germany); X. Haubois, LESIA, Observatoire de Paris à Meudon (France) and Groupement d'Intérêt Scientifique PHASE (France); T. Henning, S. Hippler, Max-Planck-Institut für Astronomie (Germany); R. Hofmann, Max-Planck-Institut für extraterrestrische Physik (Germany); L. Jocou, Lab. d'Astrophysique de l'Observatoire de Grenoble (France); S. Kellner, Max-Planck-Institut für extraterrestrische Physik (Germany); P. Kervella, LESIA, Observatoire de Paris à Meudon (France) and Groupement d'Intérêt Scientifique PHASE (France); R. Klein, N. Kudryavtseva, Max-Planck-Institut für Astronomie (Germany); S. Lacour, V. Lapeyrere, LESIA, Observatoire de Paris à Meudon (France) and Groupement d'Intérêt Scientifique PHASE (France); W. Laun, Max-Planck-Institut für Astronomie (Germany); P. Lena, LESIA, Observatoire de Paris à Meudon (France) and Groupement d'Intérêt Scientifique PHASE (France); R. Lenzen, Max-Planck-Institut für Astronomie (Germany); J. Lima, Univ. de Lisboa (Portugal); D. Moratschke, Univ. zu Köln (Germany); D. Moch, Max-Planck-Institut für extraterrestrische Physik (Germany); T. Moulin, Lab. d'Astrophysique de l'Observatoire de Grenoble (France); V. Naranjo, U. Neumann, Max-Planck-Institut für Astronomie (Germany); A. Nolot, Lab. d'Astrophysique de I'Observatoire de Grenoble (France); T. Paumard, LESIA, Observatoire de Paris à Meudon (France) and Groupement d'Intérêt Scientifique PHASE (France); O. Pfuhl, S. Rabien, Max-Planck-Institut für extraterrestrische Physik (Germany); J. Ramos, Max-Planck-Institut für Astronomie (Germany); J. M. Rees, LESIA, Observatoire de Paris à Meudon (France) and Groupement d'Intérêt Scientifique PHASE (France); R.-R. Rohloff, Max-Planck-Institut für Astronomie (Germany); D. Rouan, G. Rousset, A. Sevin, LESIA, Observatoire de Paris à Meudon (France) and Groupement d'Intérêt Scientifique PHASE (France); M. Thiel, Max-Planck-Institut für extraterrestrische Physik (Germany); K. Wagner, Max-Planck-Institut für Astronomie (Germany); M. Wiest, S. Yazici, Univ. zu Köln (Germany); D. Ziegler, LESIA, Observatoire de Paris à Meudon (France) and Groupement d'Intérêt Scientifique PHASE (France)

773411 Science with the Keck Interferometer ASTRA program [7734-36]

J. A. Eisner, Steward Observatory, The Univ. of Arizona (United States); R. Akeson, NASA Exoplanet Science Institute (United States); M. Colavita, Jet Propulsion Lab. (United States); A. Ghez, Univ. of California, Los Angeles (United States); J. Graham, Univ. of California, Berkeley (United States); L. Hillenbrand, R. Millan-Gabet, NASA Exoplanet Science Institute (United States); J. D. Monnier, Univ. of Michigan (United States); J.-U. Pott, Max-Planck-Institut für Astronomie (Germany); S. Ragland, P. Wizinowich, J. Woillez, W. M. Keck Observatory (United States)

\section{SESSION 9 FACILITIES-FUTURE: GROUND II}

773412 ASTRA: astrometry and phase-referencing astronomy on the Keck Interferometer [7734-37] J. Woillez, W. M. Keck Observatory (United States); R. Akeson, NASA Exoplanet Science Institute (United States); M. Colavita, Jet Propulsion Lab. (United States); J. Eisner, The Univ. of Arizona (United States); A. Ghez, Univ. of California, Los Angeles (United States); J. Graham, Univ. of California Berkeley (United States); L. Hillenbrand, California Institute of Technology (United States); R. Millan-Gabet, NASA Exoplanet Science Institute (United States); J. Monnier, Univ. of Michigan (United States); J.-U. Pott, Max-Planck-Institut für Astronomie (Germany); S. Ragland, P. Wizinowich, E. Appleby, B. Berkey, A. Cooper, W. M. Keck Observatory (United States); C. Felizardo, J. Herstein, NASA Exoplanet Science Institute 
(United States); M. Hrynevych, O. Martin, D. Medeiros, D. Morrison, T. Panteleeva, B. Smith, K. Summers, K. Tsubota, C. Tyau, E. Wetherell, W. M. Keck Observatory (United States)

773413 Magdalena Ridge Interferometer: assembly, integration and testing of the unit telescopes [7734-38]

O. Pirnay, M. Pierard, V. Moreau, P. Verheyden, AMOS s.a. (Belgium); C. Mayer, Observatory Sciences Ltd. (United Kingdom)

773414 Magdalena Ridge Observatory Interferometer automated alignment system [7734-39] A. V. Shtromberg, C. A. Jurgenson, K. M. McCord, A. M. Olivares, H. N. Bloemhard, F. G. Santoro, New Mexico Institute of Mining and Technology, Magdalena Ridge Observatory (United States); D. F. Buscher, C. A. Haniff, J. S. Young, Univ. of Cambridge (United Kingdom); N. C. Torres, A. R. Farris, New Mexico Institute of Mining and Technology, Magdalena Ridge Observatory (United States)

773415 The GRAVITY acquisition and guiding system [7734-40]

A. Amorim, J. Lima, Univ. de Lisboa (Portugal); O. Pfuhl, F. Eisenhaver, S. Kellner, M. Haug, M. Thiel, Max-Planck-Institut für extraterrestrische Physik (Germany); P. Carvas, Univ. de Lisboa (Portugal); G. Perrin, Observatoire de Paris à Meudon (France); W. Brandner, Max-Planck-Institut für Astronomie (Germany); C. Straubmeier, Univ. zu Köln (Germany); J.-P. Berger, Lab. d'Astrophysique de l'Observatoire de Grenoble (France)

\section{SESSION 10 OBSERVING TECHNIQUES II}

773417 The Fomalhaut debris disk seen from every angle with interferometry [7734-42] O. Absil, Univ. of Liege (Belgium); B. Mennesson, Jet Propulsion Lab. (United States); J.-B. Le Bouquin, J.-C. Augereau, LAOG, CNRS, Univ. Joseph Fourier (France); R. Millan-Gabet, M. Colavita, Jet Propulsion Lab, (United States); P. Hinz, W. Liu, Steward Observatory, The Univ. of Arizona (United States); G. Serabyn, Jet Propulsion Lab. (United States)

773418 Image reconstruction in optical interferometry: applications to the inner regions of protoplanetary disks [7734-43]

S. Renard, F. Malbet, Lab. dAstrophysique de Grenoble, CNRS, UJF (France); M. Benisty, INAF, Osservatorio Astrofisico di Arcetri (Italy); E. Thiébaut, Ctr. de Recherche Astrophysique de Lyon, CNRS, UCBL, ENSL (France); J.-P. Berger, Lab. dAstrophysique de Grenoble, CNRS, UJF (France) and European Southern Observatory (Chile)

773419 PSF and field of view characteristics of imaging and nulling interferometers [7734-44] F. Hénault, CNRS H. Fizeau, UNS, Observatoire de la Côte d'Azur (France)

7734 1A MIRC closure phase studies for high precision measurements [7734-45] M. Zhao, Jet Propulsion Lab. (United States); J. D. Monnier, X. Che, Univ. of Michigan (United States); T. ten Brummelaar, Georgia State Univ. (United States); E. Pedretti, N. D. Thureau, Univ. of St. Andrews (United Kingdom)

7734 IB Speckle imaging with the SOAR and the very large telescopes [7734-46]

S. Rengaswamy, J. H. Girard, G. Montagnier, European Southern Observatory (Chile) 
7734 1C Stellar intensity interferometry: imaging capabilities of air Cherenkov telescope arrays [7734-47]

P. D. Nuñez, S. LeBohec, D. Kieda, The Univ. of Utah (United States); R. Holmes, Boeing LTS, Inc. (United States); H. Jensen, D. Dravins, Lund Observatory (Sweden)

7734 1D Stellar intensity interferometry: experimental steps toward long-baseline observations [7734-48]

S. LeBohec, B. Adams, The Univ. of Utah (United States); I. Bond, S. Bradbury, Univ. of Leeds (United Kingdom); D. Dravins, H. Jensen, Lund Observatory (Sweden); D. B. Kieda, D. Kress, E. Munford, P. D. Nuñez, R. Price, The Univ. of Utah (United States); E. Ribak, Technion-Israel Institute of Technology (Israel); J. Rose, Univ. of Leeds (United Kingdom); H. Simpson, J. Smith, The Univ. of Utah (United States)

$7734 \mathrm{IE} \quad$ The potential of rotating-baseline nulling interferometers operating within large single-telescope apertures [7734-49]

E. Serabyn, B. Mennesson, S. Martin, K. Liewer, D. Mawet, Jet Propulsion Lab. (United States); C. Hanot, Univ. de Liège (Belgium); F. Loya, M. M. Colavita, Jet Propulsion Lab. (United States); S. Ragland, W. M. Keck Observatory (United States)

7734 1G Direct imaging with a hypertelescope of red supergiant stellar surfaces [7734-51] F. Patru, European Southern Observatory (Chile); A. Chiavassa, Max Planck Institute for Astrophysics (Germany); D. Mourard, N. Tarmoul, Lab. Fizeau (France)

\section{SESSION 12 SPACE INTERFEROMETER TECHNOLOGIES I}

$7734 \mathrm{1H} \quad$ Systems engineering and application of system performance modeling in SIM Lite mission [7734-52]

M. Moshir, D. W. Murphy, D. L. Meier, M. H. Milman, Jet Propulsion Lab. (United States)

$773411 \quad$ On-orbit dynamics and controls system architecture for SIM Lite [7734-53] O. S. Alvarez-Salazar, Jet Propulsion Lab. (United States)

7734 1J SIM Lite mission spectral calibration sensitivities and refinements [7734-54] C. Zhai, X. An, R. Goullioud, B. Nemati, M. Shao, J. Shen, U. Wehmeier, X. Wang, M. Weilert T. Werne, J. Wu, Jet Propulsion Lab. (United States)

7734 1K SIM Lite: ground alignment of the instrument [7734-55] F. G. Dekens, R. Goullioud, F. Nicaise, G. Kuan, M. Morales, Jet Propulsion Lab. (United States)

7734 IL SIM Lite Guide-2 telescope system identification, control design and pointing performance evaluation [7734-56] J. F. Shields, D. Boussalis, N. Fathpour, M. Weilert, I. Hahn, A. Ahmed, Jet Propulsion Lab. (United States) 
7734 1M The SIM Lite Astrometric Observatory: engineering risk reduction activity [7734-62]

R. Goullioud, F. Dekens, B. Nemati, X. An, L. Hovland, Jet Propulsion Lab. (United States)

7734 iN SIM interferometer testbed (SCDU) status and recent results [7734-57]

B. Nemati, X. An, R. Goullioud, M. Shao, T.-P. Shen, U. J. Wehmeier, M. A. Weilert, X. Wang,

T. A. Werne, J. P. Wu, C. Zhai, Jet Propulsion Lab. (United States)

773410 Flight qualification and performance testing of SIM precision optical mechanisms [7734-58]

A. Toorian, R. Smythe, M. Morales, J. Carson, J. D. Moore, Jet Propulsion Lab. (United States)

7734 IP Photonic technologies for a pupil remapping interferometer [7734-59]

P. Tuthill, The Univ. of Sydney (Australia); N. Jovanovic, Macquarie Univ. (Australia) and Australian Astronomical Observatory (Australia); S. Lacour, Observatoire de Paris à Meudon (France); A. Lehmann, Macquarie Univ. (Australia); M. Ams, G. Marshall, Macquarie Univ. (Australia) and Ctr. for Ultrahigh bandwidth Devices for Optical Systems (Australia); J. Lawrence, Macquarie Univ. (Australia) and Australian Astronomical Observatory (Australia); M. Withford, Macquarie Univ. (Australia) and Ctr. for Ultrahigh bandwidth Devices for Optical Systems (Australia); G. Robertson, M. Ireland, B. Pope, P. Stewart, The Univ. of Sydney (Australia)

7734 IR Picometer stable scan mechanism for gravitational wave detection in space [7734-61] N. Rijnveld, J. A. C. M. Pijnenburg, TNO Science \& Industry (Netherlands)

SESSION 14 CURRENT AND PLANNED FACILITIES

7734 is MATISSE cold optics opto-mechanical design [7734-63]

N. Tromp, F. Rigal, E. Elswijk, G. Kroes, NOVA-ASTRON (Netherlands); Y. Bresson, Observatoire de la Côte d'Azur (France); R. Navarro, NOVA-ASTRON (Netherlands)

7734 IT Stellar intensity interferometry: optimizing air Cherenkov telescope array layouts [7734-64] H. Jensen, D. Dravins, Lund Observatory (Sweden); S. LeBohec, P. D. Nuñez, The Univ. of Utah (United States)

$77341 \mathrm{U}$ LINC-NIRVANA piston control elements [7734-65]

M. Brix, J.-U. Pott, T. Bertram, Max-Planck-Institut für Astronomie (Germany); S. Rost, Univ. zu Köln (Germany); J. L. Borelli, T. M. Herbst, M. Kuerster, R.-R. Rohloff, Max-Planck-Institut für Astronomie (Germany)

7734 IV The LINC-NIRVANA fringe and flexure tracker: control design overview [7734-66] S. Rost, A. Eckart, M. Horrobin, B. Lindhorst, U. Lindhorst, L. Moser, S. Smajic, C. Straubmeier, E. Tremou, I. Wank, J. Zuther, Univ. zu Köln (Germany); T. Bertram, Max-Planck-Institut für Astronomie (Germany)

\section{SESSION 15 CRITICAL SUBSYSTEMS I}

7734 IW Testing and alignment of the LBTI [7734-67]

J. Kim, P. Hinz, O. Durney, T. Connors, M. Montoya, Steward Observatory, The Univ. of Arizona (United States); C. Schwab, Univ. Heidelberg (Germany) 
$77341 \mathrm{X} \quad$ Fringe detection and piston variability in LINC-NIRVANA [7734-68]

M. Horrobin, A. Eckart, B. Lindhorst, U. Lindhorst, L. Moser, S. Rost, S. Smajic, C. Straubmeier, E. Tremou, I. Wank, J. Zuther, Univ. zu Köln (Germany); T. Bertram, Max Planck Institute for Astronomy (Germany); C. Arcidiacono, Osservatorio Astrofisico di Arcetri (USA)

\section{SESSION 16 CRITICAL SUBSYSTEMS II}

773412 GRAVITY: design and performance of the fringe tracker [7734-70]

E. Choquet, LESIA, Observatoire de Paris à Meudon (France) and Groupement d'intérêt Scientifique PHASE (France); F. Cassaing, ONERA/DOTA (France) and Groupement d'intérêt Scientifique PHASE (France); G. Perrin, LESIA, Observatoire de Paris à Meudon (France) and Groupement d'intérêt Scientifique PHASE (France); F. Eisenhaver, Max-Planck-Institut für extraterrestrische Physik (Germany); W. Brandner, Max-Planck-Institut für Astronomie (Germany); C. Straubmeier, Univ. zu Köln (Germany); K. Perraut, Lab. d'Astrophysique, Observatoire de Grenoble (France); S. Gillessen, Max-Planck-Institut für extraterrestrische Physik (Germany)

773420 First results using PRIMA FSU as a fringe tracker for MIDI [7734-130]

A. Müller, European Southern Observatory (Germany) and Max-Planck-Institut für Astronomie (Germany); J.-U. Pott, Max-Planck-Institut für Astronomie (Germany); S. Morel, European Southern Observatory (Chile); R. Abuter, G. van Belle, European Southern Observatory (Germany); R. van Boekel, L. Burtscher, Max-Planck-Institut für Astronomie (Germany); F. Delplancke, European Southern Observatory (Germany); T. Henning, Max-Planck-Institut für Astronomie (United States); W. Jaffe, Sterrewacht Leiden, Leiden Univ. (United States); C. Leinert, Max-Planck-Institut für Astronomie (Germany); B. Lopez, A. Matter, Lab. Fizeau (France); K. Meisenheimer, Max-Planck-Institut für Astronomie (Germany); C. Schmid, European Southern Observatory (Germany); K. Tristram, Max-Planck-Institut für Radioastronomie (Germany); A. P. Verhoeff, Astronomical Institute Anton Pannekoek, Univ. of Amsterdam (Netherlands)

773421 The fringe detection laser metrology for the GRAVITY interferometer at the VLII [7734-72] H. Bartko, S. Gillessen, S. Rabien, M. Thiel, A. Gräter, M. Haug, S. Kellner, F. Eisenhaver, Max-Planck-Institut für extraterrestrische Physik (Germany); S. Lacour, LESIA, Observatoire de Paris, CNRS, UPMC, Univ. Paris Diderot (France); C. Straubmeier, Univ. zu Köln (Germany); J.-P. Berger, Lab. d'Astrophysique de l'Observatoire de Grenoble (France) and European Southern Observatory (Germany); L. Jocou, Lab. d'Astrophysique de l'Observatoire de Grenoble (France); W. Chibani, S. Lust, D. Moch, O. Pfuhl, W. Fabian, Max-Planck-Institut für extraterrestrische Physik (Germany); C. Araujo-Hauck, Univ. zu Köln (Germany); K. Perraut, Lab. d'Astrophysique de l'Observatoire de Grenoble (France); W. Brandner, Max-Planck-Institut für Astronomie (Germany); G. Perrin, LESIA, Observatoire de Paris, CNRS, UPMC, Univ. Paris Diderot (France); A. Amorim, Univ. de Lisboa (Portugal)

773422 First results from fringe tracking with the PRIMA fringe sensor unit [7734-73] J. Sahlmann, Observatoire de Genève (Switzerland), European Southern Observatory (Germany), European Southern Observatory (Chile); R. Abuter, S. Ménardi, C. Schmid, N. Di Lieto, F. Delplancke, R. Frahm, N. Gomes, European Southern Observatory (Germany); P. Haguenauer, European Southern Observatory (Chile); S. Lévêque, European Southern Observatory (Germany); S. Morel, European Southern Observatory (Chile); A. Müller, European Southern Observatory (Germany) and Max-Planck-Institut fur Astronomie 
(Germany); T. Phan Duc, European Southern Observatory (Germany); N. Schuhler, European Southern Observatory (Chile); G. van Belle, European Southern Observatory (Germany)

773423 The polarization-based collimated beam combiner and the proposed NOVA fringe tracker (NFT) for the VLTI [7734-74]

J. A. Meisner, W. J. Jaffe, Leiden Univ. (Netherlands); R. S. Le Poole, Leiden Univ. (Netherlands) and TNO Science and Industry (Netherlands); S. F. Pereira, Technische Univ. Delft (Netherlands); A. Quirrenbach, Landessternwarte Heidelberg (Germany); D. Raban, A. Vosteen, TNO Science and Industry (Netherlands)

773424 The planar optics phase sensor: a study for the VLTI 2nd generation fringe tracker [7734-76] N. Blind, J.-B. Le Bouquin, Lab. d'Astrophysique de Grenoble (France); O. Absil, Univ. de Liège (Belgium); M. Alamir, GIPSA-Lab (France); J.-P. Berger, European Southern Observatory (Chile); D. Defrère, Univ. de Liège (Belgium); P. Feautrier, Lab. d'Astrophysique de Grenoble (France); F. Hénault, Lab. H. FIZEAU, Observatoire de la Côte d'Azur (France); L. Jocou, P. Kern, Lab. d'Astrophysique de Grenoble (France); T. Laurent, Univ. de Liège (Belgium); F. Malbet, Lab. d'Astrophysique de Grenoble (France); D. Mourard, Lab. H. FIZEAU, Observatoire de la Côte d'Azur (France); K. Rousselet-Perraut, Lab. d'Astrophysique de Grenoble (France); A. Sarlette, J. Surdej, Univ. de Liège (Belgium); N. Tarmoul, Lab. H. FIZEAU, Observatoire de la Côte d'Azur (France); E. Tatulli, Lab. d'Astrophysique de Grenoble (France); L. Vincent, Lab. d'Astrophysique de Grenoble (France) and GIPSA-Lab (France)

773425 Multi-axial integrated optics solution for POPS, a 2nd-generation VLTI fringe tracker [7734-77]

N. Tarmoul, F. Hénault, D. Mourard, Lab. H. FIZEAU, CNRS, Univ. de Nice Sophia, Observatoire de la Côte d'Azur (France); J.-B. Le Bouquin, L. Jocou, Univ. Joseph-Fourier, CNRS, Lab. d'Astrophysique de Grenoble (France); P. Kern, Lab. d'Astrophysique de l'Observatoire de Grenoble (France); J.-P. Berger, European Southern Observatory (Chile); O. Absil, Univ. de Liège (Belgium)

773426 Coherent integration: to real time or not to real time? That is the question. [7734-78] A. M. Jorgensen, New Mexico Institute of Mining and Technology, Magdalena Ridge Observatory (United States); D. Mozurkewich, Seabrook Engineering (United States)

\section{Part Two}

SESSION 18 TECHNOLOGIES I

773427 The 2008-2009 outburst of the young binary system Z CMa unraveled by interferometry with high spectral resolution [7734-79]

F. Malbet, Lab. d'Astrophysique de Grenoble, Univ. Joseph Fourier, CNRS (France);

M. Benisty, INAF, Osservatorio Astrofisico di Arcetri (Italy); C. Dougados, Lab. d'Astrophysique de Grenoble, Univ. Joseph Fourier, CNRS (France); A. Natta, INAF, Osservatorio Astrofisico di Arcetri (Italy); J.-B. Le Bouquin, Lab. d'Astrophysique de Grenoble, Univ. Joseph Fourier, CNRS (France); F. Massi, INAF, Osservatorio Astrofisico di Arcetri (Italy); J. Bouvier, Lab. d'Astrophysique de Grenoble, Univ. Joseph Fourier, CNRS (France); K. Grankin, Crimean 
Astrophysical Observatory (Ukraine); M. Bonnefoy, E. Whelan, Lab. d'Astrophysique de Grenoble, Univ. Joseph Fourier, CNRS (France)

773428 Implementation of the chromatic phase diversity method on the SIRIUS test bench [7734-80] N. Tarmoul, D. Mourard, F. Hénault, J.-M. Clausse, P. Girard, A. Marcotto, N. Mauclert, A. Spang, Y. Rabbia, A. Roussel, Lab. H. FIZEAU, CNRS, Univ. de Nice Sophia Antipolis, Observatoire de la Côte d'Azur (France)

773429 Wavefront calibration and correction of an optical train path: a compliant static deformable mirror approach [7734-81]

J. H. Clark III, U.S. Naval Research Lab. (United States); F. E. Penado, Northern Arizona Univ. (United States); F. Cornelius, Lowell Observatory (United States)

7734 2A The Fiber Coupler subsystem of the future VLTI instrument GRAVITY [7734-82]

O. Pfuhl, F. Eisenhauer, M. Haug, M. Thiel, S. Kellner, Max-Planck-Institut für extraterrestrische Physik (Germany); A. Amorim, SIM, Fac. de Ciências da Univ. de Lisboa (Portugal); W. Brandner, Max-Planck-Institut für Astronomie (Germany); J.-P. Berger, Laboratoire d'Astrophysique Observatoire de Grenoble (France) and European Southern Observatory (Germany); K. Rousselet-Perraut, Laboratoire d'Astrophysique Obs. de Grenoble (France); G. S. Perrin, LESIA, Observatoire de Paris Meudon (France) and Groupement d'Intérêt Scientifique PHASE (France); C. Straubmeier, Univ. zu Köln (Germany); S. Gillessen, H. Bartko, A. P. Gräter, Max-Planck-Institut für extraterrestrische Physik (Germany)

7734 2B Reliable optical pump architecture for highly coherent lasers used in space metrology applications [7734-83]

H. Erlig, Y. Qiu, I. Poberezhskiy, P. Meras, J. Wu, Jet Propulsion Lab. (United States)

7734 2C 'OHANA-Iki: a test-bed for the 'OHANA beam combiner and delay line at CFHT [7734-84] M. Baril, O. Lai, G. Zahariade, F. Bouchacourt, Canada-France-Hawaii Telescope (United States); G. Perrin, P. Fedou, LESIA, Observatoire de Paris à Meudon (France); J. Woillez, W. M. Keck Observatory (United States)

\section{SESSION 19 TECHNOLOGIES II}

$77342 \mathrm{D} \quad$ Recent progress in wide-field imaging interferometry [7734-85]

S. A. Rinehart, D. T. Leisawitz, M. R. Bolcar, K. M. Chaprnka, R. G. Lyon, NASA Goddard Space Flight Ctr. (United States); S. F. Maher, Science Systems and Applications, Inc. (United States); N. Memarsadeghi, NASA Goddard Space Flight Ctr. (United States); E. J. Sinukoff, McMaster Univ. (Canada); E. Teichman, Univ. of Maryland, College Park (United States)

$77342 \mathrm{E}$ The NULLTIMATE test bench: achromatic phase shifters for nulling interferometry [7734-86] P. A. Schuller, O. Demangeon, A. Léger, Univ. Paris-Sud, Institut d'Astrophysique Spatiale, CNRS (France); M. Barillot, Thales Alenia Space (France); B. Chazelas, Univ. of Geneva (Switzerland); M. Decaudin, Univ. Paris-Sud, Institut d'Astrophysique Spatiale, CNRS (France); M. Derrien, Pôle Optique Rhône-Alpes (France); P. Duret, P. Gabor, Univ. Paris-Sud, Institut d'Astrophysique Spatiale, CNRS (France); G. Gadret, Lab. Interdisciplinaire Carnot de Bourgogne, CNRS, Univ. de Bourgogne (France); J. Gay, H. Fizeau, Univ. Nice Sophia Antipolis, CNRS, Observatoire de la Côte d'Azur (France); A. Labèque, Univ. Paris-Sud, Institut d'Astrophysique Spatiale, CNRS (France); R. Launhardt, Max-Planck-Institut für Astronomie (Germany); J. Mangin, Lab. Interdisciplinaire Carnot de Bourgogne, CNRS, Univ. de Bourgogne (France); Y. Rabbia, H. Fizeau, Univ. Nice Sophia Antipolis, CNRS, Observatoire 
de la Côte d'Azur (France); Z. Sodnik, European Space Research and Technology Ctr. (Netherlands)

\section{SESSION 20 SOFTWARE I}

$77342 \mathrm{~F} \quad$ Estimating the phase in interferomety: performance comparison between multi-mode and single-mode schemes [7734-87]

E. Tatulli, N. Blind, F. Malbet, A. Chelli, Lab. d'Astrophysique de l'Observatoire de Grenoble (France); J.-P. Berger, European Southern Observatory (Chile)

$77342 \mathrm{G} \quad$ Analysis of LBT LINC-NIRVANA simulated images of galaxies [7734-88]

P. Ciliegi, INAF - Osservatorio Astronomico di Bologna (Italy); A. La Camera, Univ. degli Studi di Genova (Italy); C. Arcidiacono, INAF - Arcetri Astronomical Observatory (Italy); M. Bertero, P. Boccacci, Univ. degli Studi di Genova (Italy); E. Diolaiti, INAF - Osservatorio Astronomico di Bologna (Italy); I. Foppiani, Univ. degli Studi di Bologna (Italy); M. Lombini, L. Schreiber, INAF - Osservatorio Astronomico di Bologna (Italy)

$77342 \mathrm{H} \quad$ A novel imaging algorithm for broadband aperture synthesis data [7734-89]

C. A. Hummel, European Southern Observatory (Germany)

\section{SESSION 21 SOFTWARE II}

$773421 \quad$ A novel image reconstruction software for optical/infrared interferometry [7734-90] F. Baron, J. D. Monnier, Univ. of Michigan (United States); B. Kloppenborg, Univ. of Denver (United States)

$77342 \mathrm{~J}$ Spectral regularization and sparse representation bases for interferometric imaging [7734-91]

M. Vannier, D. Mary, Lab. H. Fizeau, Univ. de Nice Sophia Antipolis (France); F. Millour, Max Planck Institute for Radio Astronomy, Bonn (Germany) and Lab. H. Fizeau, Univ. de Nice Sophia Antipolis (France); R. G. Petrov, Lab. H. Fizeau, Univ. de Nice Sophia Antipolis (France); S. Bourguignon, Cassiopé, Observatoire de la Cote d'Azur (France); C. Theys, Lab. H. Fizeau, Univ. de Nice Sophia Antipolis (France)

\section{SESSION 22 SPACE INTERFEROMETER TECHNOLOGIES III}

7734 2K New concept for direct detection and spectra of exoplanets [7734-92]

T. Matsuo, National Astronomical Observatory of Japan (Japan) and Jet Propulsion Lab. (United States); W. A. Traub, Jet Propulsion Lab. (United States); M. Hattori, Tohoku Univ. (Japan); M. Tamura, National Astronomical Observatory of Japan (Japan); M. Shao, Jet Propulsion Lab. (United States)

$77342 \mathrm{~L}$ Development of a CELestial Infrared Nuller Experiment (CELINE) for broadband nulling and new single-mode fiber testing [7734-163]

C. Hanot, P. Riaud, Univ. de Liège (Belgium); D. Mawet, Jet Propulsion Lab. (United States); O. Absil, J. Surdej, Univ. de Liège (Belgium); S. Habraken, Univ. de Liège (Belgium) and Ctr. Spatial de Liege (Belgium) 
7734 2M PERSEE: experimental results on the cophased nulling bench [7734-95]

J. Lozi, F. Cassaing, ONERA (France) and Groupement d'Intérêt Scientifique PHASE (France);

J. M. Le Duigou, Ctr. National d'Études Spatiales (France); K. Houairi, B. Sorrente, J. Montri, ONERA (France) and Groupement d'Intérêt Scientifique PHASE (France); S. Jacquinod, Institut d'Astrophysique Spatiale d'Orsay (France); J.-M. Rees, L. Pham, E. Lhome, T. Buey, Observatoire de Paris à Meudon (France) and Groupement d'Intérêt Scientifique PHASE (France); F. Hénault, A. Marcotto, P. Girard, N. Mauclert, Observatoire de la Côte d'Azur (France); M. Barillot, Thales Alenia Space (France); V. Coudé du Foresto, Observatoire de Paris à Meudon (France) and Groupement d'Intérêt Scientifique PHASE (France); M. Ollivier, Institut d'Astrophysique Spatiale d'Orsay (France)

\section{SESSION 23 OBSERVING TECHNOLOGIES}

$77342 \mathrm{~N}$ The 2010 interferometric imaging beauty contest [7734-96]

F. Malbet, Lab. d'Astrophysique de Grenoble, Univ. J. Fourier, CNRS (France) and Jean-Marie Mariotti Ctr. (France); W. Cotton, National Radio Astronomy Observatory (United States); G. Duvert, Lab. d'Astrophysique de Grenoble, Univ. J. Fourier, CNRS (France) and Jean-Marie Mariotti Ctr. (France); P. Lawson, Jet Propulsion Lab. (United States); A. Chiavassa, Max-Planck-Institut für Astrophysik (Germany); J. Young, Cavendish Lab. (United Kingdom); F. Baron, Univ. of Michigan (United States); D. Buscher, Cavendish Lab. (United Kingdom); S. Rengaswamy, European Southern Observatory (Germany); B. Kloppenborg, Univ. of Denver (United States); M. Vannier, Lab. H. Fizeau, Univ. de Nice-Sophia Antipolis (France); L. Mugnier, ONERA, DOTA (France)

773420 Optical Long Baseline Interferometry News (OLBIN) [7734-97] P. R. Lawson, Jet Propulsion Lab. (United States); F. Malbet, Lab. d'Astrophysique de l'Observatoire de Grenoble (France)

7734 2P Developing achromatic coronagraphic optics for LMIRCam and the LBT [7734-98] M. A. Kenworthy, Leiden Observatory, Leiden Univ. (Netherlands) and Steward Observatory, The Univ. of Arizona (United States); P. M. Hinz, J. L. Codona, Leiden Observatory, Leiden Univ. (United States); J. C. Wilson, M. F. Skrutskie, Univ. of Virginia (United States); E. Solheid, Leiden Observatory, Leiden Univ. (United States)

$77342 \mathrm{Q}$ Coherent integration results from the NPOI [7734-99]

A. M. Jorgensen, New Mexico Institute of Mining and Technology, Magdalena Ridge Observatory (United States); H. R. Schmitt, U.S. Naval Research Lab. (United States) and Computational Physics, Inc. (United States); J. T. Armstrong, U.S. Naval Research Lab. (United States); D. Mozurkewich, Seabrook Engineering (United States); E. K. Baines, R. Hindsley, U.S. Naval Research Lab. (United States); D. Hutter, U.S. Naval Observatory (United States); S. Restaino, U.S. Naval Research Lab. (United States)

$77342 R \quad$ Toward the stability required for direct observations of exoplanets with nulling interferometry [7734-100]

O. Demangeon, P. A. Schuller, A. Léger, P. Duret, Univ. Paris-Sud, Institut d'Astrophysique Spatiale, CNRS (France)

773425 Development of a statistical reduction method for the Palomar Fiber Nuller [7734-101] C. Hanot, Univ. de Liège (Belgium); B. Mennesson, E. Serabyn, S. Martin, K. Liewer, F. Loya, D. Mawet, Jet Propulsion Lab. (United States); P. Riaud, O. Absil, Univ. de Liège (Belgium) 
$77342 \mathrm{~T} \quad$ Integrated optic beam combiners for stellar interferometry and nulling at near- and mid-infrared wavelengths [7734-102]

H. Hsiao, K. A. Winick, J. D. Monnier, Univ. of Michigan (United States); J.-P. Berger, Lab.

d'Astrophysique, CNRS, Observatoire de Grenoble (France)

POSTER SESSION: CURRENT AND PLANNED FACILITIES AND INSTRUMENTS

$77342 \mathrm{U} \quad$ Review of OCA activities on nulling testbench PERSEE [7734-103]

F. Hénault, CNRS H. Fizeau, UNS, Observatoire de la Côte d'Azur (France); P. Girard, Observatoire de la Côte d'Azur (France); A. Marcotto, N. Mauclert, C. Bailet, J.-M. Clausse,

D. Mourard, Y. Rabbia, A. Roussel, CNRS H. Fizeau, UNS, Observatoire de la Côte d'Azur (France); M. Barillot, Thales Alenia Space (France); F. Cassaing, ONERA (France); J.-M. Le Duigou, Ctr. National d'Études Spatiales (France)

$77342 \mathrm{~V} \quad$ High precision interferometer: MIRC with photometric channels [7734-104]

X. Che, J. D. Monnier, S. Webster, Univ. of Michigan (United States)

7734 2W Measuring the effective wavelength of CHARA classic [7734-105]

E. C. Bowsher, H. A. McAlister, Georgia State Univ. (United States); T. A. ten Brummelaar, The CHARA Array, Mount Wilson Observatory (United States)

$77342 X \quad$ The LINC-NIRVANA fringe and flexure tracker: first measurements of the testbed interferometer [7734-106]

L. Moser, Univ. zu Köln (Germany); A. Eckart, Univ. zu Köln (Germany) and Max-Planck-Institu† für Radioastronomie (Germany); M. Horrobin, B. Lindhorst, S. Rost, C. Straubmeier, E. Tremou, I. Wank, J. Zuther, Univ. zu Köln (Germany); T. Bertram, Max-Planck-Institut für Astronomie (Germany)

$77342 Y \quad$ OVMS: the optical path difference and vibration monitoring system for the LBT and its interferometers [7734-107]

M. Kürster, T. Bertram, J. L. Borelli, M. Brix, W. Gässler, T. M. Herbst, V. Naranjo, J.-U. Pott, J. Trowitzsch, Max-Planck-Institut für Astronomie (Germany); T. E. Connors, P. M. Hinz, T. J. McMahon, Steward Observatory, The Univ. of Arizona (United States); D. S. Ashby, J. G. Brynnel, N. J. Cushing, T. Edgin, J. D. Esguerra, R. F. Green, J. Kraus, J. Little, LBTO, The Univ. of Arizona (United States); U. Beckmann, G. P. Weigelt, Max-Planck-Institut für Radioastronomie (Germany)

$77342 Z$ GRAVITY spectrometer: metrology laser blocking strategy at OD=12 [7734-108]

C. Araujo-Hauck, S. Fischer, Univ. zu Köln (Germany); H. Bartko, S. Gillessen, Max-Planck-Institut für extraterrestrische Physik (Germany); C. Straubmeier, M. Wiest, S. Yazici, Univ. zu Köln (Germany); F. Eisenhaver, Max-Planck-Institut für extraterrestrische Physik (Germany); G. S. Perrin, Lab. d'Etudes Spatiales et d'Instumentation en Astrophysique (France) and Institut National des Sciences de I'Univers (France); W. Brandner,

Max-Planck-Institut für Astronomie (Germany); K. Perraut, Lab. d'Astrophysique Observatoire de Grenoble (France); A. Amorim, Facultade de Ciencias da Univ. de Lisboa (Portugal);

A. Eckart, Univ. zu Köln (Germany) and Max Planck Institute für Radio Astronomie (Germany)

773430 The GRAVITY integrated optics beam combination [7734-109]

L. Jocou, K. Perraut, A. Nolot, LAOG, CNRS (France); J.-P. Berger, European Southern Observatory (Germany); T. Moulin, LAOG, CNRS (United States); P. Labeye, CEA/LETI (France); S. Lacour, G. Perrin, Max-Planck-Institute for extraterrestrial physics (Germany); 
J. B. Lebouquin, LAOG, CNRS (France); H. Bartko, M. Thiel, F. Eisenhaver, LESIA, Observatoire de Paris à Meudon (France)

773432 The GRAVITY spectrometers: optical design and principle of operation [7734-111] C. Straubmeier, S. Fischer, C. Araujo-Hauck, M. Wiest, S. Yazici, Univ. zu Köln (Germany); F. Eisenhaver, Max-Planck-Institut für extraterrestrische Physik (Germany); G. Perrin, LESIA, Observatoire de Paris, CNRS, UPMC (France), Institut National des Sciences de I'Univers (France), and Groupement d'Interet Scientifique PHASE (France); W. Brandner, Max-Planck-Institut für Astronomie (Germany); K. Perraut, Lab. d'Astrophysique Observatoire Grenoble (France); A. Amorim, Faculdade de Ciencias da Univ. de Lisboa (Portugal); M. Schöller, European Southern Observatory (Germany); A. Eckart, Univ. zu Köln (Germany) and Max-Planck-Institut fur Radioastronomie (Germany)

773434 The hydrogen emission of young stellar objects: key science for next-generation instruments and facilities [7734-113]

P. J. V. Garcia, Univ. Joseph Fourier, CNRS, Institut de Planetologie et d'Astrophysique de Grenoble (Portugal) and Univ. do Porto (Portugal); M. Benisty, S. Rajabi, INAF, Osservatorio Astrofisico di Arcetri (Italy); C. Dougados, Univ. Joseph Fourier, CNRS, Institut de Planetologie et d'Astrophysique de Grenoble (France); F. Massi, F. Bacciotti, INAF, Osservatorio Astrofisico di Arcetri (Italy); J.-B. Le Bouquin, F. Malbet, Univ. Joseph Fourier, CNRS, Institut de Planetologie et d'Astrophysique de Grenoble (France); L. Podio, Kapteyn Astronomical Institute (Netherlands); S. Renard, E. Whelan, Univ. Joseph Fourier, CNRS, Institut de Planetologie et d'Astrophysique de Grenoble (France)

773435 PIONIER: a visitor instrument for VLII [7734-1 14]

J.-P. Berger, LAOG, UJF, CNRS (France) and European Southern Observatory (Chile); G. Zins, B. Lazareff, J.-B. Lebouquin, L. Jocou, P. Kern, LAOG, UJF, CNRS (France); R. Millan-Gabet, NASA Exoplanet Science Institute (United States); W. Traub, Jet Propulsion Lab. (United States); P. Haguenauer, European Southern Observatory (Chile); O. Absil, Univ. de Liege (Belgium); J. Augereau, LAOG, UJF, CNRS (France); M. Benisty, INAF, Osservatorio Astrofisico di Arcetri (Italy); N. Blind, X. Bonfils, A. Delboulbe, P. Feautrier, M. Germain, Lab.

d'Astrophysique de Grenoble (Chile); D. Gillier, Lab. d'Astrophysique de l'Observatoire de Grenoble (France); P. Gitton, European Southern Observatory (Chile); M. Kiekebusch, J. Knudstrup, J. Lizon, European Southern Observatory (Germany); Y. Magnard, F. Malbet, D. Maurel, F. Menard, M. Micallef, L. Michaud, LAOG, UJF, CNRS (France); S. Morel, European Southern Observatory (Chile); T. Moulin, LAOG, UJF, CNRS (France); D. Popovic, European Soutnern Observatory (Chile); K. Perraut, P. Rabou, S. Rochat, F. Roussel, A. Roux, E. Stadler, E. Tatulli, LAOG, UJF, CNRS (France)

POSTER SESSION: FACILITY ISSUES

773436 Evaluation of performance of the MACAO systems at the VLTI [7734-115]

S. Rengaswamy, P. Haguenauer, S. Brillant, A. Cortes, J. H. Girard, European Southern Observatory (Chile); S. Guisard, J. Paufique, European Southern Observatory (Germany); A. Pino, European Southern Observatory (Chile) 
773437 Status of the VLTI-UT performances wrt vibrations [7734-116]

S. Poupar, P. Haguenauer, A. Merand, J. Alonso, P. Bourget, S. Brillant, R. Castillo, N. Di Lieto, European Southern Observatory (Chile); J.-L. Lizon, European Southern Observatory (Germany); P. Gitton, European Southern Observatory (Chile); J. Sahlmann, Univ. de Genève (Switzerland); N. Schuhler, European Southern Observatory (Chile)

773438 Comparison between closure phase and phase referenced interferometric image reconstructions [7734-117]

N. Gomes, European Organisation for Astronomical Research in the Southern Hemisphere (Germany) and Univ. do Porto (Portugal) and Lab. de Sistemas (Portugal); P. J. V. Garcia, Lab. d'Astrophysique (France) and Lab. d'Astrophysique de l'Observatoire de Grenoble (Portugal); E. M. Thiébaut, Observatoire de Lyon (France); S. Renard, Lab. d'Astrophysique de l'Observatoire de Grenoble (France); M. Filho, Centro de Astrofísica da Univ. do Porto (Portugal)

773439 The effects of atmospheric calibration errors on source model parameters [7734-118] C. Tycner, Central Michigan Univ. (United States); D. J. Hutter, R. T. Zavala, U. S. Naval Observatory (United States)

7734 3A Dual three-way infrared beam combiner at the CHARA Array [7734-119] J. Sturmann, T. ten Brummelaar, L. Sturmann, H. A. McAlister, Georgia State Univ. (United States)

7734 3C Detection of a geostationary satellite with the Navy Prototype Optical Interferometer [7734-121]

J. T. Armstrong, R. B. Hindsley, U.S. Naval Research Lab. (United States); H. R. Schmitt, Computational Physics, Inc. (United States); F. J. Vrba, J. A. Benson, D. J. Hutter, R. T. Zavala, U.S. Naval Observatory (United States)

7734 3D Imaging simulations of selected science with the Magdalena Ridge Observatory Interferometer [7734-122]

M. Creech-Eakman, New Mexico Institute of Mining and Technology, Magdalena Ridge Observatory (United States); J. Young, C. Haniff, D. Buscher, Univ. of Cambridge (United Kingdom); M. Elvis, Harvard-Smithsonian Ctr. for Astrophysics (United States); A. Chiavassa, Max Planck Institute for Astrophysics (Germany); M. Schartmann, Max Planck Institute for Extraterrestrial Physics (Germany)

7734 3F A publication database for optical long baseline interferometry [7734-124] F. Malbet, G. Mella, Lab. d'Astrophysique de Grenoble, Univ. J. Fourier, CNRS (France) and Jean-Marie Mariotti Ctr. (France); P. Lawson, Jet Propulsion Lab. (United States); E. Taillifet, S. Lafrasse, Lab. d'Astrophysique de Grenoble, Univ. J. Fourier, CNRS (France) and Jean-Marie Mariotti Ctr. (France)

$77343 G$ Observations of binaries with the NPOI [7734-125]

H. R. Schmitt, Computational Physics, Inc. (United States) and U.S. Naval Research Lab. (United States); J. T. Armstrong, U.S. Naval Research Lab. (United States); A. M. Jorgensen, New Mexico Institute of Mining and Technology, Magdalena Ridge Observatory (United States); E. K. Baines, R. B. Hindsley, U.S. Naval Research Lab. (United States) 
7734 3H SIM Lite detection of habitable planets in P-type binary-planetary systems [7734-126] X. Pan, M. Shao, S. Shaklan, R. Goullioud, Jet Propulsion Lab. (United States)

$773431 \quad$ Homothetic apodization of circular aperture HACA: simulation results [7734-127]

O. Azagrouze, A. Habib, Y. Elazhari, Z. Benkhaldoun, M. Lazrek, Cadi Ayyad Univ. (Morocco)

7734 3J Tunable spatial heterodyne spectroscopy (TSHS): a new technique for broadband visible interferometry [7734-128]

S. S. Hosseini, A. Gong, D. Ruth, H. A. Baldis, W. Harris, Univ. of California, Davis (United States)

7734 3K From fringes to the USNO Navy Prototype Optical Interferometer Astrometric Catalog [7734-129]

J. A. Benson, D. J. Hutter, R. T. Zavala, H. C. Harris, P. D. Shankland, K. J. Johnston, U.S. Naval Observatory (United States)

7734 3L Limb-darkened angular diameters of stars with combined infrared and optical interferometry [7734-131]

E. K. Baines, J. T. Armstrong, U.S. Naval Research Lab. (United States); H. R. Schmitt, U.S. Naval Research Lab. (United States) and Computational Physics, Inc. (United States)

\section{POSTER SESSION: TECHNOLOGIES}

$77343 \mathrm{M} \quad$ A very wide-field wavefront sensor for a very narrow-field interferometer [7734-132] V. Viotto, INAF, Osservatorio Astronomico di Padova (Italy) and Univ. di Padova (Italy); R. Ragazzoni, INAF, Osservatorio Astronomico di Padova (Italy); C. Arcidiacono, INAF, Osservatorio Astronomico di Firenze (Italy); M. Bergomi, Univ. di Padova (Italy); A. Brunelli, Univ. degli Studi di Padova (Italy); M. Dima, J. Farinato, G. Gentile, D. Magrin, INAF, Osservatorio Astronomico di Padova (Italy); G. Cosentino, Univ. di Bologna (Italy); E. Diolaiti, I. Foppiani, M. Lombini, INAF, Osservatorio Astronomico di Bologna (Italy); L. Schreiber, Univ. di Bologna (Italy); T. Bertram, P. Bizenberger, F. De Bonis, W. Gässler, T. Herbst, M. Kuerster, D. Meschke, L. Mohr, R.-R. Rohloff, Max-Planck-Institut für Astronomie (Germany)

$77343 \mathrm{~N}$ Monolithic Michelson Interferometer as ultra stable wavelength reference [7734-133] X. Wan, J. Ge, Univ. of Florida (United States)

773430 Development of a high-dynamic range imaging instrument for a single telescope by a pupil remapping system [7734-134]

T. Kotani, ISAS/JAXA (Japan); S. Lacour, E. Choquet, E. Huby, G. S. Perrin, P. Fedou, Observatoire de Paris à Meudon (France); F. Marchis, SETI Institute (United States) and Univ. of Califolnia, Berkeley (United States); G. Duchene, Univ. of Califolnia, Berkeley (United States) and Lab. d'Astrophysique de l'Observatoire de Grenoble (France); É. M. Thiébaut, Ctr. de Recherche Astrophysique de Lyon, CRAL, CNRS (France); J. M. Woillez, W. M. Keck Observatory (United States); J.-P. Berger, Lab. d'Astrophysique de l'Observatoire de Grenoble (France); P. J. Bordé, Institut d'Astrophysique Spatiale, Univ. Paris-Sud 11 (France); O. Chesneau, Observatoire de la Côte d'Azur (France); P. Kervella, Observatoire de Paris à Meudon (France); O. Lai, Canada-France-Hawaii Telescope (United States); S. T. Ridgway, Kitt Peak National Observatory (United States); D. Rouan, Observatoire de Paris à Meudon (France); A. Lecavelier des Ełangs, A. Vidal-Madjar, Institut d'Astrophysique de Paris, CNRS, Univ. Pierre \& Marie Curie (France) 
7734 3P Three-dimensional photonic combiner for optical astro interferometry [7734-136]

S. Minardi, T. Pertsch, R. Neuhäuser, Friedrich-Schiller-Univ. Jena (Germany)

7734 3Q Development of Monolithic Michelson Interferometer for RV measurement in IR [7734-137] J. Wang, X. Wan, J. C. Ge, Univ. of Florida (United States)

$77343 R \quad$ Single-mode mid-infrared waveguides for spectro-interferometry applications [7734-138] G. Martin, Lab. d'Astrophysique de l'Observatoire de Grenoble (France); L. Labadie, Instituto de Astrofísica de Canarias (Spain); O. Caballero-Calero, R. Grille, B. Arezki, P. Kern, Lab. d'Astrophysique de l'Observatoire de Grenoble (France); N. C. Anheier, H. A. Qiao, B. Bernacki, Pacific Northwest National Lab. (United States); T. Lewi, A. Katzir, Tel Aviv Univ. (Israel); J. Rodriguez Vazquez de Aldana, Univ. de Salamanca (Spain)

773435 Feeding the wavefront sensors of LINC-NIRVANA: the dedicated Patrol Camera [7734-139] D. Lorenzetti, F. D'Alessio, G. Li Causi, F. Vitali, M. Centrone, R. Speziali, NAF, Osservatorio Astronomico di Roma (Italy); E. Diolaiti, INAF, Osservatorio Astronomico di Bologna (Italy); J. Farinato, INAF, R. Ragazzoni, INAF, Osservatorio Astronomico di Padova (Italy); T. Bertram, F. Briegel, F. De Bonis, W. Gässler, T. Herbst, M. Kürster, R. Rohloff, Max-Planck-Institut für Astronomie (Germany)

7734 3T A new embedded control system for SUSI [7734-140] W. J. Tango, M. J. Ireland, The Univ. of Sydney (Australia)

$77343 \mathrm{U}$ Custom beamsplitter and AR coatings for interferometry [7734-141] K. Nyland, C. A. Jurgenson, New Mexico Institute of Mining and Technology, Magdalena Ridge Observatory (United States); D. F. Buscher, C. A. Haniff, J. S. Young, Univ. of Cambridge (United Kingdom); J. Lewis, R. Schnell, Optical Surface Technologies, LLC (United States)

7734 3V Fringe modulation for an MROI beam combiner [7734-142] T. M. McCracken, C. A. Jurgenson, New Mexico Institute of Mining and Technology, Magdalena Ridge Observatory (United States); D. H. Baird, David Baird Embedded Systems (United States); J. K. Seamons, K. M. McCord, New Mexico Institute of Mining and Technology (United States); D. F. Buscher, C. A. Haniff, J. S. Young, Univ. of Cambridge (United Kingdom)

7734 3W Fresnel diffraction in an interferometer: application to MATISSE [7734-143] S. Robbe-Dubois, Y. Bresson, E. Aristidi, S. Lagarde, P. Antonelli, B. Lopez, R. G. Petrov, Univ. de Nice-Sophia antipolis, Observatoire de la Côte d'Azur, CNRS (France)

7734 3X Hybrid sol-gel technology for fast prototyping in astronomical interferometry [7734-144] A. Ghasempour, Univ. do Porto (Portugal), XLIM Institut de Recherche (France), and Tennessee State Univ. (United States); A. M. P. Leite, Univ. do Porto (Portugal);

D. Alexandre, Univ. do Porto (Portugal), INESC Porto (Portugal), and Univ. de Trás-os-Montes e Alto Douro (Portugal); F. Reynaud, XLIM Institut de Recherche (France); P. V. S. Marques, Univ. do Porto (Portugal) and INESC Porto (Portugal); P. J. V. Garcia, Univ. do Porto (Portugal) 
7734 3Y MAMMUT: mirror vibration metrology for VLTI [7734-145]

I. Spaleniak, F. Gießler, R. Geiss, S. Minardi, T. Pertsch, R. Neuhäuser, Friedrich-Schiller-Univ. Jena (Germany); M. Becker, M. Rothhardt, IPHT Jena (Germany); F. Delplancke, A. Richichi,

S. Ménardi, C. Schmid, European Southern Observatory (Germany)

\section{POSTER SESSION: CRITICAL SUBSYSTEMS}

$77343 Z$ The atmospheric piston simulator as an integral part of the calibration unit of LINC-NIRVANA [7734-146]

R. Follert, T. M. Herbst, P. Bizenberger, F. DeBonis, Max-Planck-Institut für Astronomie (Germany)

773441 The LINC-NIRVANA fringe and flexure tracker: laboratory tests [7734-148]

E. Tremou, A. Eckart, Univ. zu Köln (Germany) and Max-Planck-Institut for Radioastronomy (Germany); M. Horrobin, B. Lindhorst, L. Moser, S. Rost, S. Smajic, C. Straubmeier, I. Wank,

J. Zuther, Univ. zu Köln (Germany); T. Bertram, Max-Planck-Institut für Astronomie (Germany)

773443 Beam combination with a large number of apertures [7734-150]

D. Mozurkewich, Seabrook Engineering (United States); A. Traore, Sigma Space Corp. (United States)

773444 Mechanical design of the Magdalena Ridge Observatory Interferometer [7734-151]

F. G. Santoro, A. M. Olivares, C. D. Salcido, S. R. Jimenez, New Mexico Institute of Mining and Technology, Magdalena Ridge Observatory (United States); X. Sun, C. A. Haniff,

D. F. Buscher, Univ. of Cambridge (United Kingdom); M. J. Creech-Eakman, C. A. Jurgenson, A. V. Shtromberg, E. J. Bakker, R. J. Selina, New Mexico Institute of Mining and Technology (United States); M. Fisher, J. S. Young, D. M. A. Wilson, Univ. of Cambridge (United Kingdom)

773445 Modified telescope alignment procedure for improving the beam quality of the CHARA Telescopes [7734-152]

L. Sturmann, J. Sturmann, T. A. ten Brummelaar, H. A. McAlister, Georgia State Univ. (United States)

773446 The MROI fringe tracker: first fringe experiment [7734-153]

C. Jurgenson, F. Santoro, T. McCracken, K. McCord, A. Shtromberg, D. Klinglesmith,

A. Olivarez, New Mexico Institute of Mining and Technology, Magdalena Ridge Observatory (United States); D. Buscher, Univ. of Cambridge (United Kingdom); M. Creech-Eakman, New Mexico Institute of Mining and Technology (United States); C. Haniff, J. Young, Univ. of Cambridge (United Kingdom)

773447 A new control architecture for multi-beam fringe tracker [7734-154]

L. Vincent, Univ. of Grenoble (France) and Lab. d'Astrophysique de Grenoble, CNRS, Univ. Joseph Fourier (France); M. Alamir, Univ. of Grenoble (France); J.-B. Le Bouquin, European Southern Observatory (Chile); L. Jocou, K. Rousselet-Perraut, P. Kern, J.-P. Berger, Lab. d'Astrophysique de Grenoble, CNRS, Univ. Joseph Fourier (France)

773448 The LINC-NIRVANA fringe and flexure tracker: an update of the opto-mechanical system [7734-155]

J. Zuther, A. Eckart, Univ. zu Köln (Germany); T. Bertram, Max-Planck-Institut für Astronomie (Germany); M. Horrobin, B. Lindhorst, U. Lindhorst, L. Moser, S. Rost, C. Straubmeier, E. Tremou, I. Wank, Univ. zu Köln (Germany) 
773449 Design of the MROI delay line optical path compensator [7734-156]

M. Fisher, R. C. Boysen, D. F. Buscher, C. A. Haniff, E. B. Seneta, X. Sun, D. M. A. Wilson,

J. S. Young, Univ. of Cambridge (United Kingdom)

\section{POSTER SESSION: SOFTWARE}

7734 4A Bias-free imaging at low light levels [7734-157]

J. Gordon, D. Buscher, H. Thorsteinsson, Univ. of Cambridge (United Kingdom)

7734 4B The data-reduction software for micro-arcsecond astrometry with PRIMA at the VLTI [7734-158]

R. Köhler, I. Stilz, Zentrum für Astronomie der Univ. Heidelberg (Germany) and Max-Planck-Institut für Astronomie (Germany); A. Quirrenbach, A. Kaminski, Zentrum für Astronomie der Univ. Heidelberg (Germany); T. Schulze-Hartung, R. Launhardt, Max-Planck-Institut für Astronomie (Germany); N. M. Elias II, National Radio Astronomy Observatory (United States); T. Henning, Max-Planck-Institut für Astronomie (Germany); D. Queloz, Univ. of Geneva (Switzerland)

7734 4C The third version of the AMBER data reduction software [7734-159]

F. Malbet, G. Duvert, Lab. d'Astrophysique de Grenoble, Univ. Joseph Fourier, CNRS (France); F. Millour, Max-Planck-Institut für Radioastronomie (Germany); J.-B. Le Bouquin, G. Mella, L. Halipré, A. Chelli, S. Lafrasse, E. Altariba, G. Zins, Lab. d'Astrophysique de Grenoble, Univ. Joseph Fourier, CNRS (France)

7734 4D GPU-accelerated image reconstruction for optical and infrared interferometry [7734-160] F. Baron, Univ. of Michigan (United States); B. Kloppenborg, Univ. of Denver (United States)

7734 4E Building the 'JMMC Stellar Diameters Catalog' using SearchCal [7734-161]

S. Lafrasse, G. Mella, Univ. Joseph Fourier, CNRS, Lab. d'Astrophysique de Grenoble (France); D. Bonneau, Univ. Nice Sophia Antipolis, CNRS, Observatoire de la Côte d'Azur (France); G. Duvert, X. Delfosse, Univ. Joseph Fourier, CNRS, Lab. d'Astrophysique de Grenoble (France); O. Chesneau, Univ. Nice Sophia Antipolis, CNRS, Observatoire de la Côte d'Azur (France); A. Chelli, Univ. Joseph Fourier, CNRS, Lab. d'Astrophysique de Grenoble (France)

7734 4F SCDU testbed automated in-situ alignment, data acquisition, and analysis [7734-162] T. A. Werne, U. J. Wehmeier, J. P. Wu, X. An, R. Goullioud, B. Nemati, M. Shao, T.-P. J. Shen, X. Wang, M. A. Weilert, C. Zhai, Jet Propulsion Lab. (United States)

\section{POSTER SESSION: SPACE INTERFEROMETER TECHNOLOGY}

$77344 \mathrm{G}$ The fulfillment of two-level control in experimental optical delay line of Michelson Stellar Interferometer [7734-164]

L. Chao, Nanjing Institute of Astronomical Optics \& Technology (China) and Graduate Univ. of Chinese Academy of Sciences (China); W. Zhen, J. Wang, L. Yi, Y. Chen, Nanjing Institute of Astronomical Optics \& Technology (China) 
$77344 \mathrm{H} \quad$ Results of the Guide-2 telescope testbed for the SIM Light Astrometric Observatory [7734-165]

I. Hahn, M. Weilert, J. Sandhu, X. Wang, R. Smythe, E. Hovland, F. Loya, J. Shields, D. Boussalis, N. Fathpour, B. Kang, A. Ahmed, G. Macala, F. Nicaise, M. Morales, F. Dekens, R. Goullioud, Jet Propulsion Lab. (United States)

77344 Mitigation of angle tracking errors due to color dependent centroid shifts in SIM Lite [7734-166]

B. Nemati, X. An, R. Goullioud, M. Shao, T. Shen, U. J. Wehmeier, M. A. Weilert, X. Wang,

T. A. Werne, J. P. Wu, C. Zhai, Jet Propulsion Lab. (United States)

7734 4J SCDU (Spectral Calibration Development Unit) testbed narrow angle astrometric performance [7734-167]

X. Wang, R. Goullioud, B. Nemati, M. Shao, U. J. Wehmeier, M. A. Weilert, T. A. Werne, J. P. Wu,

C. Zhai, Jet Propulsion Lab. (United States)

7734 4K Progress on SIM-Lite brassboard interferometer integration and test [7734-168]

I. Hahn, M. Weilert, X. An, G. Kuan, L. Hovland, R. Smythe, E. Hovland, R. Krylo, M. Fisher, Z. Chang, J. Cepeda-Rizo, J. Shields, A. Ahmed, N. Fathpour, J. Carson, F. Nicaise, M. Morales, F. Dekens, R. Goullioud, Jet Propulsion Lab. (United States)

Author Index 


\title{
Conference Committee
}

\author{
Symposium Chairs
}

Masanori lye, National Astronomical Observatory of Japan (Japan)

Douglas A. Simons, Gemini Observatory (United States)

Symposium Cochairs

Mark M. Casali, European Organisation for Astronomical Research in the Southern Hemisphere (Germany)

Kathryn A. Flanagan, Space Telescope Science Institute (United States)

\section{Conference Chairs}

William C. Danchi, NASA Goddard Space Flight Center (United States)

Françoise Delplancke, European Organisation for Astronomical Research in the Southern Hemisphere (Germany)

Jayadev K. Rajagopal, National Optical Astronomy Observatory (United States) and Cerro Tololo Inter-American Observatory (Chile)

\section{Program Committee}

Rachel L. Akeson, California Institute of Technology (United States)

Eric J. Bakker, New Mexico Institute of Mining and Technology (United States)

Joshua A. Eisner, The University of Arizona Steward Observatory (United States)

Christopher A. Haniff, University of Cambridge (United Kingdom)

Michael J. Ireland, The University of Sydney (Australia)

Pierre Y. Kern, Laboratoire d'Astrophysique de l'Observatoire de Grenoble (France)

Bruno Lopez, Observatoire de la Côte d'Azur (France)

Harold A. McAlister, Georgia State University (United States)

Antoine Mérand, European Organisation for Astronomical Research in the Southern Hemisphere (Chile)

Markus Schoeller, European Organisation for Astronomical Research in the Southern Hemisphere (Germany)

Hiroshi Shibai, Osaka University (Japan)

Christopher Tycner, Central Michigan University (United States) 
Session Chairs

1 Current and Planned Facilities: Ground I

Eric J. Bakker, New Mexico Institute of Mining and Technology (United States)

2 Current and Planned Facilities: Ground II

Harold A. McAlister, Mount Wilson Institute (United States)

3 Current and Planned Facilities: Ground III

Rachel L. Akeson, California Institute of Technology (United States)

$4 \quad$ Current and Planned Instruments: Ground

Christopher A. Haniff, University of Cambridge (United Kingdom)

5 Current and Planned Facilities and Instruments: Space I

Joshua A. Eisner, The University of Arizona, Steward Observatory (United States)

6 Current and Planned Facilities and Instruments: Space II

Françoise Delplancke, European Organisation for Astronomical Research in the Southern Hemisphere (Germany)

7 Observing Techniques I

Christopher Tycner, Central Michigan University (United States)

8 Facilities-Future: Ground I

Markus Schoeller, European Organisation for Astronomical Research in the Southern Hemisphere (Germany)

9 Facilities-Future: Ground II

Rachel L. Akeson, California Institute of Technology (United States)

10 Observing Techniques II

William C. Danchi, NASA Goddard Space Flight Center (United States)

11 Observing Techniques III

Markus Schoeller, European Organisation for Astronomical Research in the Southern Hemisphere (Germany)

12 Space Interferometer Technologies I

Pierre Y. Kern, Laboratoire d'Astrophysique de l'Observatoire de Grenoble (France)

13 Space Interferometer Technologies II

Christopher Tycner, Central Michigan University (United States) 
14 Current and Planned Facilities

Markus Schoeller, European Organisation for Astronomical Research in the Southern Hemisphere (Germany)

15 Critical Subsystems I

Markus Schoeller, European Organisation for Astronomical Research in the Southern Hemisphere (Germany)

16 Critical Subsystems II

Eric J. Bakker, New Mexico Institute of Mining and Technology (United States)

17 Critical Subsystems III

Christopher Tycner, Central Michigan University (United States)

18 Technologies I

Antoine Mérand, European Organisation for Astronomical Research in the Southern Hemisphere (Chile)

19 Technologies II

Joshua A. Eisner, The University of Arizona, Steward Observatory (United States)

20 Software I

Joshua A. Eisner, The University of Arizona, Steward Observatory (United States)

21 Software II

Françoise Delplancke, European Organisation for Astronomical Research in the Southern Hemisphere (Germany)

22 Space Interferometer Technologies III

Françoise Delplancke, European Organisation for Astronomical Research in the Southern Hemisphere (Germany)

23 Observing Technologies

William C. Danchi, NASA Goddard Space Flight Center (United States) 
Downloaded From: https://www.spiedigitallibrary.org/conference-proceedings-of-spie on 25 Apr 2023

Terms of Use: https://www.spiedigitallibrary.org/terms-of-use 